[16] Movérare, R., Westritschnig, K., Svensson, M., Hayek, B., Bende, M., Pauli, G. et. al. (2002). Different IgE Reactivity Profiles in Birch Pollen-Sensitive Patients from Six European Populations Revealed by Recombinant Allergens: An Imprint of Local Sensitization. International Archives of Allergy and Immunology, 128 (4), 325-335. doi: http://doi.org/10.1159/000063855

[17] Westman, M., Lupinek, C., Bousquet, J., Andersson, N., Pahr, S., Baar, A. et. al. (2015). Early childhood IgE reactivity to pathogenesis-related class 10 proteins predicts allergic rhinitis in adolescence. Journal of Allergy and Clinical Immunology, 135 (5), 1199-1206. doi: http://doi.org/10.1016/ j.jaci.2014.10.042

[18] McKenna, O. E., Asam, C., Araujo, G. R., Roulias, A., Goulart, L. R., Ferreira, F. (2016). How relevant is panallergen sensitization in the development of allergies? Pediatric Allergy and Immunology, 27 (6), 560-568. doi: http://doi.org/10.1111/pai.12589

[19] Macchia, D., Melioli, G., Pravettoni, V., Nucera, E., Piantanida, M. et. al. (2015). Guidelines for the use and interpretation of diagnostic methods in adult food allergy. Clinical and Molecular Allergy, 13 (1). doi: http://doi.org/10.1186/s12948-015-0033-9

[20] Hauser, M., Roulias, A., Ferreira, F., Egger, M. (2010). Panallergens and their impact on the allergic patient. Allergy, Asthma \& Clinical Immunology, 6 (1). doi: http://doi.org/10.1186/1710-1492-6-1

[21] Wolthers, O. D. (2012). Component-Resolved Diagnosis in Pediatrics. ISRN Pediatrics, 2012, 1-6. doi: http://doi.org/10.5402/2012/806920

[22] Deng, S., Yin, J. (2019). Mugwort Pollen-Related Food Allergy: Lipid Transfer Protein Sensitization and Correlation With the Severity of Allergic Reactions in a Chinese Population. Allergy, Asthma \& Immunology Research, 11 (1), 116-128. doi: http://oi.org/10.4168/aair.2019.11.1.116

[23] San Nicoló, M., Braun, T., Eder, K., Berghaus, A., Gröger, M. (2016). Clinical Relevance of IgE to Profilin and/or Polcalcin in Pollen-Sensitized Patients. International Archives of Allergy and Immunology, 169 (2), 101-107. doi: http://doi.org/10.1159/000444279

\title{
EVALUATION OF CENTRIFUGING REGIMES FOR THE PURPOSE OF OPTIMIZING THE PLATELET RICH PLASMA HARVESTING PROTOCOL
}

\author{
Sergiy Chetverikov \\ Surgery department No. 4 with oncology course \\ Odessa National Medical University \\ 2 Valichovskiy lane, Odessa, Ukraine, 65082 \\ Dmitro Atanasov \\ Surgery department No. 4 with oncology course \\ Odessa National Medical University \\ 2 Valichovskiy lane, Odessa, Ukraine, 65082 \\ dmitriyatanasov@gmail.com
}

\footnotetext{
Abstract

Aim: Based on the classical principles, to determine the optimal conditions for centrifugation, PRP harvesing (platelet-rich plasma). To conduct a quantitative assessment of the substrate obtained under different conditions of centrifugation.

Materials and methods. Based on the basic principles of obtaining platelet-rich plasma (PRP) by centrifuging in containers with an anticoagulant followed by phase separation to obtain the final substrate, the efficiency of the technique under the conditions of single and double centrifugation as well as under different conditions of acceleration and centrifugation was evaluated.

Blood for follow-up was collected from 20 healthy volunteers (11 men, 9 women) average $25.3 \pm 4.1$ in syringes of LuerLock design with ACD-A anticoagulant solution, and centrifuged. Centrifugation was carried out under controlled conditions using a centrifuge with rotating bowls of the rotor. Centrifugation was performed at an acceleration of $100-400 \mathrm{~g}$ in time intervals up to 20 minutes. Activation of the substrate was performed with calcium chloride solution.

Quantitative evaluation of platelets of whole blood and the final substrate of PRP was carried out with a semi-automatic analyzer.
} 
Results. The obtained results demonstrate the maximum level of harvesting efficiency when performing double centrifugation in the $150 \mathrm{~g} \times 15 \mathrm{~min}+250 \mathrm{~g} \times 10 \mathrm{~min}$ mode. Subject to this centrifugation protocol, it is possible to obtain a substrate that complies with the standardized requirements for PRP.

The maximum level of an increase in the number of platelets in the substrate in comparison with whole blood is determined at the level of $\times 4.36$ with concentration (volume reduction) $\times 5$ in comparison with the volume of whole blood.

Conclusions. This study demonstrated the advantage of double centrifuging modes over single modes.

According to the results of the study, it was possible to determine the conditions for an optimal double-centrifugation mode (acceleration and duration), which allows us to achieve the most efficient concentration of the substrate.

Keywords: platelet rich plasma, platelets, red blood cells, centrifugation, syringe, harvesing, acceleration.

\section{Introduction}

Platelet-rich plasma platelet-based products (platelets-rich plasma, PRP) have been widely introduced into clinical practice due to the pronounced stimulating effect of the substrate $[1,2]$. The autologous nature of the substrate is important, which almost completely eliminates the risk of immunological reactions and infectious and transplant complications [3, 4]. The high efficacy as well as the safety of platelet-rich plasma products (PRP) ensured its considerable popularity in dental surgery, as evidenced by significant number of publications in this field [5, 6]. Also, the enormous amount of work demonstrates the effectiveness of plasma-rich platelets in orthopedics for sports injuries [7, 8]. In plastic, reconstructive surgery, cosmetology, PRP is applicable for the purpose of correcting skin changes, including number and age $[9,10]$, as well as modifications of ischemic tissues during procedures related to the transplantation of flaps, fat graft $[11,12]$. Also products based on PRP are used in the practice of cardiovascular, neurosurgeons, urologists, ginecologists $[13,14]$. Despite the fact that the safety of the substrate does not cause doubts according to the overwhelming majority of authors, the question remains about the observed lack of effectiveness as well as varying degrees of severity of the stimulating effect in solving similar problems according to various authors $[15,16]$. A significant number of ready-made solutions proposed by manufacturers for obtaining plasma enriched with platelets, according to the results of application, according to the instructions, does not correspond to the expected declared results, which is stated in the works of some authors $[17,18]$. Thus at this stage it is impossible to single out a single optimal method of harvesting as the gold standard for the implementation of the PRP procedure [19].

According to the classical principles of the procedure for obtaining platelets-rich plasma: the patient's whole blood is collected in a container with an anticoagulant, which is subsequently subjected to centrifugation [20]. Various containers are available on the market with differing principles of phase separation after centrifugation. At the same time, with similar designs of test tubes, containers, different manufacturers recommended different centrifuging modes: at different acceleration, of different duration. In some cases, one-step centrifugation is recommended, which contradicts other recommendations stating the higher efficiency of the double centrifugation mode [19, 21].

Kits with a gel separator of fractions of the RegenLab type (Switzerland) are difficult to work with, have a significant drawback associated with the high cost of consumables, and also demonstrate low efficiency in quantifying the final substrate. Another option for PRP production systems is kits with containers of an improved design, which simplifies the procedure for selecting separated phases, thus, to a lesser extent, the procedure depends on the skills of the contractor, but often such solutions are expensive. A more common option is kits based on classic PRGF EndoRet test tubes (PlasmaRichinGrowthFactors) from Biotechnology Institute, Spain, but this technology has the disadvantage of certain skill requirements due to the complexity of manual phase selection after centrifugation. Also applicable are techniques based on the use of containers that are securely and hermetically sealed for injection with the subsequent selection of the separated phases by moving between the syringes. Such harvesting options are highly cost-effective, but require specific skills from the performer [20, 22].

In all versions, the main stage of harvesting will be the procedure of phase separation (erythrocytes, thrombocytic layer, and plasma) by centrifugation. Determination of optimal conditions: 
acceleration, centrifugation duration as well as comparison of two-stage and one-stage centrifugation in harvesting remain an urgent problem, which does not have an unambiguous universal solution. The determination of optimal conditions is especially important in centrifuging techniques using syringe containers.

\section{Aim of the work}

Determine the optimal conditions for centrifuging whole blood in order to obtain the production of plasma enriched with platelets using a syringe container.

\section{Materials and methods}

The study was performed on the basis of the Center for Reconstructive and Reconstructive Medicine as well as the Department of Surgery No. 4 with a course of oncology at the Odessa National Medical University in 2018-2019.

Blood for the study was collected from 20 healthy volunteers (11 men, 9 women) with an average age of $25.3 \pm 4.1$. All volunteers did not complain and did not suffer from chronic diseases. The blood of each of the volunteers was collected in 4 syringes with a volume of $5 \mathrm{ml}$ in which $0.5 \mathrm{ml}(10 \%$ of the volume) of the anticoagulant CITRAT glucose solution A (ACD-A) was previously collected. In all cases, identical syringes with a LuerLock design connector were used. Syringes were closed with a Kombi-stopper congruent plug. Each syringe was adapted in size for the purpose of placing it in the rotor bowl by removing excess piston length and removing finger stops. The syringe container was placed in the thicket of the rotor and subjected to centrifugation under various conditions of acceleration and centrifugation duration. Simultaneously centrifugation of 4 blood samples from various volunteers was performed. Centrifugation was performed under strictly controlled conditions of rotational speed, centrifugal acceleration, centrifugation time using a HettichRotofix 32a centrifuge (Germany), with a symmetrical rotor, into 4 rotary bowls adapted for Arthrex ACP containers.

In the group of studies with a single centrifugation, the syringe container was removed from the rotor bowl with manual sampling $1 / 5$ the volume of the syringe $(1 \mathrm{ml})$ - including the buffer layer supplemented with the base plasma layer. The separation of the separated phases was carried out by aspirating the contents through a three-way valve into another syringe of the same volume with removal of the plasma apical layer, and preservation of the main thrombocytic-leukocyte layer with the base plasma layer. The substrate has been investigated.

In the double centrifugation study group, the first stage of centrifugation was carried out in a similar way. After aspiration, a plasma layer with a buffer (thrombocytic-leukocyte) layer was separated from the erythrocyte layer by means of aspiration through a three-way tap, Diskofix C. The syringe after the first stage of centrifugation with separated erythrocyte layer was disposed of. The syringe into which plasma aspiration was carried out with the buffer layer was closed with a Kombistoper plug and similarly adapted to the dimensions of the rotor bowl for subsequent centrifugation, was installed vertically. The result of the second centrifugation is the resultant layer at the base of the syringe container $(1 \mathrm{ml})$, which was selected by a similar procedure by aspiration through the threeway disc Diskofix, with this procedure the excess volume over $1 \mathrm{ml}$ of the resulting substrate was removed and also disposed of. The resulting substrate was examined. The strict condition was that the positioning of the syringe containers should be vertical when selecting the separated layers.

Centrifugation was performed with centrifugal acceleration of $100 \mathrm{~g}, 150 \mathrm{~g}, 200 \mathrm{~g}$ for 10 minutes, 15 minutes. Such initial parameters were determined empirically based on the experience of using the proposed technique earlier as well as data from other authors.

All studies were performed at a stable temperature of about $17{ }^{\circ} \mathrm{C}$, with a humidity of $55 \%$.

Estimation of the platelet count of whole blood and the obtained PRP substrate was carried out using a Micro-CC 20 semi-automatic hematology analyzer (HTI, USA). The following indicators were determined in all of the examined samples of whole blood and the resulting substrate; the results were analyzed: platelet count.

Statistical analysis: data were analyzed using Statistica 13.3 software. Values are expressed as mean \pm standard deviation. Analysis between groups with continuous data: single and double 
centrifugation was performed using Student's t-test and chi-square test. Pearson's correlation analysis was performed to analyze linear correlations between variables. $\mathrm{P}<0.05$ was considered statistically significant. The quantitative criteria that were determined were: PRP concentration efficiency, platelet multiplication factor. The formulas for their calculation are given below. Analyzing the concentration of erythrocytes and leukocytes in the PRP substrate, it is possible to indirectly judge the effectiveness of the separation of the buffer layer and the "purity" of the final substrate.

$$
\begin{gathered}
\text { Concentration efficiency PRP }(\%)= \\
=\frac{\text { Volume PRP }(\mathrm{ml}) \times \text { Concentration PLT PRP }\left(\times 10^{9} / \mathrm{l}\right)}{\text { Blood volume }(\mathrm{ml}) \times \text { Whole blood concentration PLT }\left(\times 10^{9} / \mathrm{l}\right)} ;
\end{gathered}
$$

$$
\begin{gathered}
\text { Platelet multiplication factor }= \\
=\frac{\text { Concentration PLT PRP }\left(\times 10^{9} / 1\right)}{\text { Whole blood concentration PLT }\left(\times 10^{9} / 1\right)} .
\end{gathered}
$$

Analyzing the concentration of erythrocytes and leukocytes, it is possible to indirectly judge the effectiveness of the separation of the buffer layer.

\section{Results}

Table 1 shows data on the subjects, demonstrated quantitative indicators of hematological analysis. Both groups of research: a group of one-stage centrifugation, a group of double centrifugation demonstrates a high level of sample homogeneity. The level of $\mathrm{P}$ value is given in the table

\begin{tabular}{|c|c|c|c|}
\hline Criterion & Single centrifugation & Double centrifugation & P criterion \\
\hline The number of studied samples & 40 & 40 & \\
\hline Gender ratio $(\mathrm{M} / \mathrm{F})$ & $22 / 18$ & $22 / 18$ & \\
\hline Age (years) & $25.3 \pm 4.1$ & $25.3 \pm 4.1$ & \\
\hline Leukocyte count $\left(\times 10^{9} / 1\right)$ & $6.37 \pm 1.67$ & $6.14 \pm 1.82$ & 0.561 \\
\hline The number of red blood cells $\left(\times 10^{12} / 1\right)$ & $4.54 \pm 0.71$ & $4.23 \pm 0.68$ & 0.826 \\
\hline Platelet Count $\left(\times 10^{9} / 1\right)$ & $228 \pm 45.4$ & $243 \pm 38.2$ & 0.694 \\
\hline
\end{tabular}
(Table 1).

Table 1

Information about the group of the studied samples (in 20 patients 80 blood samples were collected)

Platelet concentration in the final substrate of platelet-rich plasma is the main indicator of the effectiveness of harvesting. Data comparing the concentration of platelets in the resulting substrate with different modes of centrifugation are shown visually (Fig. 1). These data demonstrate the advantage of the centrifuging mode $150 \mathrm{~g} \times 15$ min with a significant $(\mathrm{p}<0.05)$ increase in the platelet level higher in comparison with the groups $100 \mathrm{~g} \times 15 \mathrm{~min}, 150 \mathrm{~g} \times 10 \mathrm{~min}, 200 \mathrm{~g} \times 10 \mathrm{~min}$, $200 \mathrm{~g} \times 15 \mathrm{~min}$. Thus, the fundamentally lower efficiency of the $100 \mathrm{~g}$ modes is demonstrated due to the fact that this acceleration under the conditions of our study demonstrated the inconsistency of concentration, namely, probably due to the insufficient difference in the sedimentation rate of the erythrocyte and buffer layers. On the other hand, an increase in centrifugation rate with an increase in centrifugal acceleration up to $200 \mathrm{~g}$ also demonstrates a slight decrease in efficiency. With a further increase in acceleration to $400 \mathrm{~g}$, we observed a significant decrease in the concentration of platelets, which is likely due to their significant damage as well as to the complex hemodynamic effects between the erythrocyte and buffer layers in conditions of excessive exposure. Thus, an increase in acceleration to $250-400 \mathrm{~g}$ at the stage of primary centrifugation is untenable. Results close to optimal $(150 \mathrm{~g} \times 15 \mathrm{~min})$ were also observed in the $150 \mathrm{~g} \times 20 \mathrm{~min}$ group; however, this 
modification, in comparison with the optimal one, carries only additional time costs without showing advantages.

Comparing the efficiency of platelet concentration in single and double centrifugation protocol, we reliably noted the advantage of the double protocol $(\mathrm{p}=0.013)$ compared to the single protocol in those modes that were rated as optimal. Differences lose statistical significance in groups of $100 \mathrm{~g}, 200 \mathrm{~g}$.

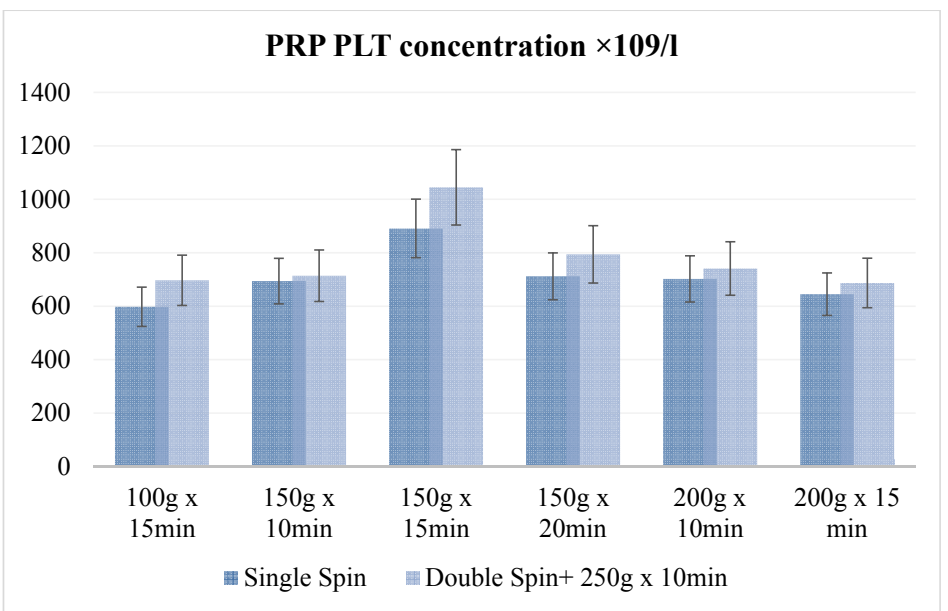

Fig. 1. Platelet concentration in the resulting PRP substrate in centrifugal modes: $100 \mathrm{~g} \times 15 \mathrm{~min}$, $150 \mathrm{~g} \times 10 \mathrm{~min}, 150 \mathrm{~g} \times 15 \mathrm{~min}, 150 \mathrm{~g} \times 20 \mathrm{~min}, 200 \mathrm{~g} \times 10 \mathrm{~min}, 200 \mathrm{~g} \times 15 \mathrm{~min}$. With single and double (with the addition of $250 \mathrm{~g} \times 10 \mathrm{~min}$ ) centrifugation

Analyzing the platelet number multiplication indicator fully confirms the results outlined above with respect to platelet concentration. This similarity of results can be explained, including the close homogeneity of the groups of studied samples. Similarly, the advantage of the $150 \mathrm{~g} \times 15 \mathrm{~min}$ protocol is demonstrated among all protocols of a single centrifugation as well as a $150 \mathrm{~g} \times 15 \mathrm{~min}+250 \mathrm{~g} \times 10 \mathrm{~min}$ protocol from the general number of observations with a reliable statistical advantage $(\mathrm{p}<0 / 05)$ (Fig. 2).

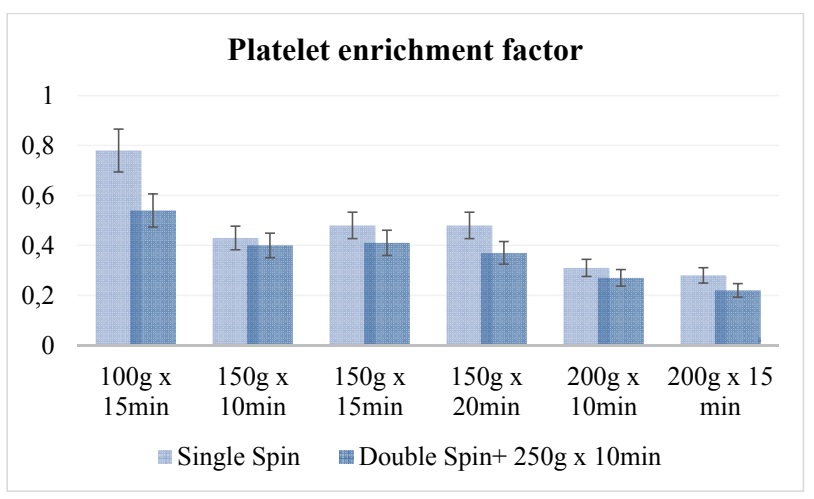

Fig. 2. Platelet multiplication factor: in the resulting PRP substrate in centrifugal modes: $100 \mathrm{~g} \times 15 \mathrm{~min}, 150 \mathrm{~g} \times 10 \mathrm{~min}, 150 \mathrm{~g} \times 15 \mathrm{~min}, 150 \mathrm{~g} \times 20 \mathrm{~min}, 200 \mathrm{~g} \times 10 \mathrm{~min}, 200 \mathrm{~g} \times 15 \mathrm{~min}$. With single and double (with the addition of $250 \mathrm{~g} \times 10 \mathrm{~min}$ ) centrifugation.

One of the available criteria for assessing the correctness of the separation of layers when performing harvesting is an estimate of the number of erythrocytes or hemoglobin level in the final PRP substrate (Fig. 3). Some authors note the high significance of this criterion, while others state its low importance, including this indicator may vary coordinately depending on the technical features of the selection procedure for the separated layers, the experience of the performer as well as the use of various systems. According to our observations, the minimum level of red blood cell 
concentration was reached in the centrifuging mode $200 \mathrm{~g} \times 10 \mathrm{~min}$. Optimal in terms of platelet count, a mode of $150 \mathrm{~g} \times 15 \mathrm{~min}+250 \mathrm{~g} \times 10$ min demonstrates significantly $(\mathrm{p}<0.05)$ a higher level of red blood cell concentration.

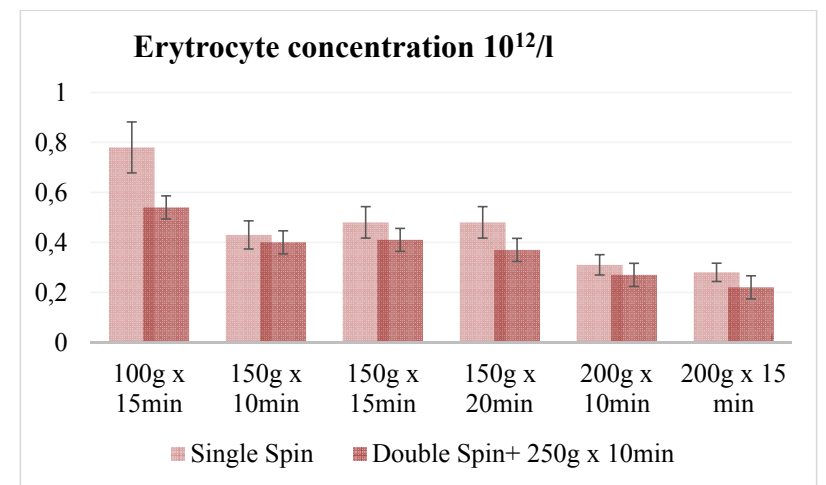

Fig. 3. RBC content in the resulting PRP substrate in centrifuging modes:

$100 \mathrm{~g} \times 15 \mathrm{~min}, 150 \mathrm{~g} \times 10 \mathrm{~min}, 150 \mathrm{~g} \times 15 \mathrm{~min}, 150 \mathrm{~g} \times 20 \mathrm{~min}, 200 \mathrm{~g} \times 10 \mathrm{~min}, 200 \mathrm{~g} \times 15 \mathrm{~min}$.

With single and double (with the addition of $250 \mathrm{~g} \times 10 \mathrm{~min}$ ) centrifugation

The concentration of leukocytes in the PRP substrate can also be regarded in two ways. According to the data of various authors, both positive and negative effects of increased leukocyte concentration in PRP are evaluated and in many respects such an assessment depends on the objectives of the use of the substrate. We can state that the level of leukocytes in the centrifuging mode of $150 \mathrm{~g} \times 15 \mathrm{~min}+250 \mathrm{~g} \times 10 \mathrm{~min}$ does not exceed the permissible limits (Fig. 4.). Also, the dynamics of the concentration of leukocytes in the comparison groups despite the statistical accuracy should not be regarded as a decisive factor in the choice of centrifugation. In our opinion, the demonstrated significantly lower concentration of leukocytes in the $200 \mathrm{~g} \times 15 \mathrm{~min}+250 \mathrm{~g} \times 10 \mathrm{~min}$ group can be decisive in choosing such a protocol only for narrowly specialized purposes, in cases where introducing a leukocyte component into PRP will have a significantly negative effect [23].

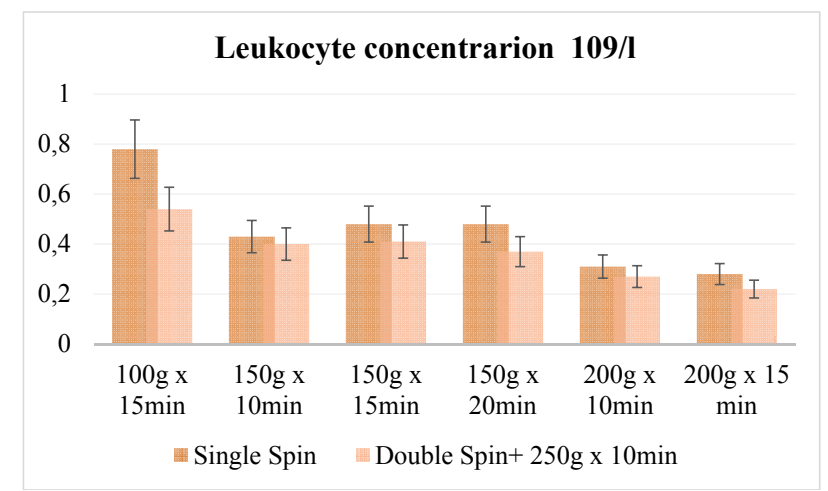

Fig. 4. The content of leukocytes in the resulting PRP substrate in centrifuging modes: $100 \mathrm{~g} \times 15 \mathrm{~min}, 150 \mathrm{~g} \times 10 \mathrm{~min}, 150 \mathrm{~g} \times 15 \mathrm{~min}, 150 \mathrm{~g} \times 20 \mathrm{~min}, 200 \mathrm{~g} \times 10 \mathrm{~min}, 200 \mathrm{~g} \times 15 \mathrm{~min}$. With single and double (with the addition of $250 \mathrm{~g} \times 10 \mathrm{~min}$ ) centrifugation

\section{Discussion}

Analyzing the data from earlier publications, it can be concluded that various authors have radically differently evaluated similar centrifugation protocols and also demonstrated radically different protocols as optimal for a harvester in completely equal conditions, which maintains the relevance and interest in this topic.

A study by Baussetetal [19] demonstrated that centrifugation at $250 \mathrm{~g} \times 15 \mathrm{~min}$, which is less commonly used today. A possible reason may be that the centrifugal conditions have a negative effect, including on platelet activity. During the first centrifugation, Baussetetal (10), namely 
$130 \mathrm{~g} \times 15 \mathrm{~min}$, shows similar data with the one proposed by us [15]. The results of this study showed that the first rotation at $150 \mathrm{~g} \times 15 \mathrm{~min}$ captured more platelets, in comparison with the control groups, which corresponds to the data of Baussetetal.

The level of erythrocytes determined by us corresponds to the permissible limits, which confirms the consistency of the technology of centrifugation in syringe containers and also the technology of selection of separated layers through aspiration with a syringe manually under visual control through a three-way valve Diskofiks $\mathrm{S}$.

The quantitative assessment of the proposed technology of centrifugation and phase selection with a certain optimal centrifugation protocol demonstrates a high level of efficiency, which is not inferior to ready-made solutions proposed on the market [24].

In our opinion, the level of red blood cells to a lesser extent affects the choice of the mode of centrifugation. A certain level of leukocytes in the resulting substrate demonstrates a significantly lower level in the conditions of centrifugation of $250 \mathrm{~g} \times 15 \mathrm{~min}$ which can be regarded as a significant factor in modifying the procedure in the case of a clinical task in which the leukocyte buffer can have a negative effect, otherwise in the majority of cases leukocyte concentration to a lesser extent affects the choice of the mode of centrifugation during harvesting.

\section{Conclusions}

1. Harvesting technique of whole blood with obtaining platelet-rich plasma demonstrated its viability with regard to the analysis of the quantitative characteristics imposed on the substrate.

2. According to the results of the study, the double centrifugation mode $150 \mathrm{~g} \times 15 \mathrm{~min}+$ $+250 \mathrm{~g} \times 10 \mathrm{~min}$ was determined as the optimal one.

3. Dual centrifugation protocol reliably demonstrated an advantage over a single one.

4. In order to minimize leukocyte buffer in the substrate, the protocol $250 \mathrm{~g} \times 15 \mathrm{~min}+$ $+250 \mathrm{~g} \times 10 \mathrm{~min}$ can be recommended.

\section{References}

[1] Liu, Y.-S., Ou, M.-E., Liu, H., Gu, M., Lv, L.-W., Fan, C. et. al. (2014). The effect of simvastatin on chemotactic capability of SDF-1 $\alpha$ and the promotion of bone regeneration. Biomaterials, 35 (15), 44894498. doi: http://doi.org/10.1016/j.biomaterials.2014.02.025

[2] Andersen, R. K., Zaher, W., Larsen, K. H., Ditzel, N., Drews, K., Wruck, W. et. al. (2015). Association between in vivo bone formation and ex vivo migratory capacity of human bone marrow stromal cells. Stem Cell Research \& Therapy, 6 (1). doi: http://doi.org/10.1186/s13287-015-0188-9

[3] Bendinelli, P., Matteucci, E., Dogliotti, G., Corsi, M. M., Banfi, G., Maroni, P., Desiderio, M. A. (2010). Molecular basis of anti-inflammatory action of platelet-rich plasma on human chondrocytes: Mechanisms of NF-kB inhibition via HGF. Journal of Cellular Physiology, 225 (3), 757-766. doi: http://doi.org/ $10.1002 /$ jcp. 22274

[4] Castillo, T. N., Pouliot, M. A., Kim, H. J., Dragoo, J. L. (2010). Comparison of Growth Factor and Platelet Concentration From Commercial Platelet-Rich Plasma Separation Systems. The American Journal of Sports Medicine, 39 (2), 266-271. doi: http://doi.org/10.1177/0363546510387517

[5] Wang, X., Zhang, Y., Choukroun, J., Ghanaati, S., Miron, R. J. (2017). Effects of an injectable platelet-rich fibrin on osteoblast behavior and bone tissue formation in comparison to platelet-rich plasma. Platelets, 29 (1), 48-55. doi: http://doi.org/10.1080/09537104.2017.1293807

[6] Kundu, R., Rathee, M. (2014). Effect of Platelet-Rich-Plasma (PRP) and Implant Surface Topography on Implant Stability and Bone. Journal of clinical and diagnostic research, 8 (6), 26-30. doi: http://doi.org/ $10.7860 / \mathrm{jcdr} / 2014 / 9177.4478$

[7] Mlynarek, R. A., Kuhn, A. W., Bedi, A. (2016). Platelet-Rich Plasma (PRP) in Orthopedic Sports Medicine. American journal of orthopedics, 45 (5), 290-326.

[8] Wasserman, A., Matthewson, G., MacDonald, P. (2018). Platelet-Rich Plasma and the KneeApplications in Orthopedic Surgery. Current Reviews in Musculoskeletal Medicine, 11 (4), 607-615. doi: http://doi.org/10.1007/s12178-018-9521-0 
[9] Cameli, N., Mariano, M., Cordone, I., Abril, E., Masi, S., Foddai, M. L. (2017). Autologous Pure Platelet-Rich Plasma Dermal Injections for Facial Skin Rejuvenation. Dermatologic Surgery, 43 (6), 826-835. doi: http://doi.org/10.1097/dss.0000000000001083

[10] Dhurat, R., Sukesh, M. (2014). Principles and methods of preparation of platelet-rich plasma: A review and author's perspective. Journal of Cutaneous and Aesthetic Surgery, 7 (4), 189-197. doi: http://doi.org/ 10.4103/0974-2077.150734

[11] Lu, J., Hao, Y., Zhao, W., Lyu, C., Zou, D. (2017). Molecular, Cellular and Pharmaceutical Aspects of Autologous Grafts for Peri-implant Hard and Soft Tissue Defects. Current Pharmaceutical Biotechnology, 18 (1), 85-94. doi: http://doi.org/10.2174/1389201017666161201165742

[12] Picard, F., Hersant, B., La Padula, S., Meningaud, J.-P. (2017). Platelet-rich plasma-enriched autologous fat graft in regenerative and aesthetic facial surgery: Technical note. Journal of Stomatology, Oral and Maxillofacial Surgery, 118 (4), 228-231. doi: http://doi.org/10.1016/j.jormas.2017.05.005

[13] Charneux, L., Demoulin, C., Vanderthomment, M., Tomasella, M., Ferrara, M.-A., Grosdent, S. et. al. (2017). Plasma riche en plaquettes (PRP) et lésions discales : revue de la littérature. Neurochirurgie, 63 (6), 473-477. doi: http://doi.org/10.1016/j.neuchi.2017.06.002

[14] Matz, E. L., Pearlman, A. M., Terlecki, R. P. (2018). Safety and feasibility of platelet rich fibrin matrix injections for treatment of common urologic conditions. Investigative and Clinical Urology, 59 (1), 61-65. doi: http://doi.org/10.4111/icu.2018.59.1.61

[15] Cavallo, C., Roffi, A., Grigolo, B., Mariani, E., Pratelli, L., Merli, G. et. al. (2016). Platelet-Rich Plasma: The Choice of Activation Method Affects the Release of Bioactive Molecules. BioMed Research International, 2016, 1-7. doi: http://doi.org/10.1155/2016/6591717

[16] Mazzocca, A. D., McCarthy, M. B. R., Chowaniec, D. M., Cote, M. P., Romeo, A. A., Bradley, J. P. et. al. (2012). Platelet-Rich Plasma Differs According to Preparation Method and Human Variability. The Journal of Bone and Joint Surgery-American Volume, 94 (4), 308-316. doi: http://doi.org/10.2106/ jbjs.k.00430

[17] Kececi, Y., Ozsu, S., Bilgir, O. (2014). A cost-effective method for obtaining standard platelet-rich plasma. WOUNDS, 26, 232-238.

[18] Castillo, T. N., Pouliot, M. A., Kim, H. J., Dragoo, J. L. (2011). Comparison of Growth Factor and Platelet Concentration From Commercial Platelet-Rich Plasma Separation Systems. The American Journal of Sports Medicine, 39 (2), 266-271. doi: http://doi.org/10.1177/0363546510387517

[19] Bausset, O., Giraudo, L., Veran, J., Magalon, J., Coudreuse, J.-M., Magalon, G. et. al. (2012). Formulation and Storage of Platelet-Rich Plasma Homemade Product. BioResearch Open Access, 1 (3), 115123. doi: http://doi.org/10.1089/biores.2012.0225

[20] Magalon, J., Bausset, O., Serratrice, N., Giraudo, L., Aboudou, H., Veran, J. et. al. (2014). Characterization and Comparison of 5 Platelet-Rich Plasma Preparations in a Single-Donor Model. Arthroscopy: The Journal of Arthroscopic \& Related Surgery, 30 (5), 629-638. doi: http://doi.org/10.1016/ j.arthro.2014.02.020

[21] Fitzpatrick, J., Bulsara, M. K., McCrory, P. R., Richardson, M. D., Zheng, M. H. (2017). Analysis of platelet-rich plasma extraction: variations in platelet and blood components between 4 common commercial kits. Orthopaedic Journal of Sports Medicine, 5 (1). doi: http://doi.org/10.1177/2325967116675272

[22] Perez, A. G. M., Lana, J. F. S. D., Rodrigues, A. A., Luzo, A. C. M., Belangero, W. D., Santana, M. H. A. (2014). Relevant Aspects of Centrifugation Step in the Preparation of Platelet-Rich Plasma. ISRN Hematology, 2014, 1-8. doi: http://doi.org/10.1155/2014/176060

[23] McCarrel, T. M., Minas, T., Fortier, L. A. (2012). Optimization of Leukocyte Concentration in Platelet-Rich Plasma for the Treatment of Tendinopathy. The Journal of Bone \& Joint Surgery, 94 (19), e143. doi: http://doi.org/10.2106/jbjs.1.00019

[24] Magalon, J., Bausset, O., Serratrice, N., Giraudo, L., Aboudou, H., Veran, J. et. al. (2014). Characterization and Comparison of 5 Platelet-Rich Plasma Preparations in a Single-Donor Model. Arthroscopy: The Journal of Arthroscopic \& Related Surgery, 30 (5), 629-638. doi: http://doi.org/10.1016/ j.arthro.2014.02.020 